\title{
Stationary Patterns for a Lotka-Volterra Cooperative Model with a Density-Dependent Diffusion Term
}

\author{
By \\ Kazuhiro OEDA \\ (Waseda University, Japan)
}

\begin{abstract}
This paper is concerned with positive stationary solutions for a LotkaVolterra cooperative model with a density-dependent diffusion term of a fractional type. The existence of stationary patterns is proven by the presence of densitydependent diffusion. Our proof is based on the Leray-Schauder degree theory and some a priori estimates. We also derive a certain limiting system which positive stationary solutions satisfy.

Key Words and Phrases. Cooperative model, Density-dependent diffusion, Stationary patterns, Leray-Schauder degree theory, Limiting system.

2000 Mathematics Subject Classification Numbers. Primary 35J65; Secondary 35B40, 35K 57, 92D25.
\end{abstract}

\section{Introduction}

In this paper we study positive stationary solutions of the following strongly coupled reaction-diffusion system:

$$
(\mathrm{P}) \begin{cases}u_{t}=\Delta\left[\left(1+\frac{\alpha}{\mu+v}\right) u\right]+u(a-u+c v) & \text { in } \Omega \times(0, T), \\ v_{t}=\Delta v+v(-b+d u-v) & \text { in } \Omega \times(0, T), \\ \frac{\partial u}{\partial n}=\frac{\partial v}{\partial n}=0 & \text { on } \partial \Omega \times(0, T), \\ u(\cdot, 0)=u_{0}(\cdot), v(\cdot, 0)=v_{0}(\cdot) & \text { in } \Omega,\end{cases}
$$

where $\Omega$ is a bounded domain in $\boldsymbol{R}^{N}$ with smooth boundary $\partial \Omega ; \partial / \partial n$ denotes the outward normal derivative on $\partial \Omega ; a, b, c, d$ and $\mu$ are all positive constants; $\alpha$ is a non-negative constant; $u_{0}$ and $v_{0}$ are given non-negative functions which are not identically zero. The system $(\mathrm{P})$ is a Lotka-Volterra cooperative model with a density-dependent diffusion term of a fractional type; unknown functions $u$ and $v$ represent population densities of two cooperative species, respectively; $a$ and $-b$ denote the intrinsic growth rates of the respective species; $c$ and $d$ denote interaction coefficients. When $\alpha=0,(\mathrm{P})$ is reduced to a classical LotkaVolterra cooperative model with diffusion. See [9] and [15] for such a cooperative model. 
According to Okubo and Levin [13], a typical diffusion term appearing in the theory of population dynamics is represented in the form of $\Delta[D(x, t) w]$ where $w$ denotes a population density and $D(x, t)$ reflects the transition probability of individuals of species $w$ at a position $x$ and time $t$. We call $D(x, t)$ a diffusion coefficient. In certain cooperative relationships in ecology, the movement of one species depends on the population density of the other species. In such a situation, the diffusion coefficient of one species will be large (resp. small) at a point where the population density of the other species is low (resp. high). Taking the above points into consideration, we take $1+\alpha /(\mu+v)$ as the diffusion coefficient for $u$ in $(\mathrm{P})$. The nonlinear diffusion term $\alpha \Delta[u /(\mu+v)]$ will play the most important role in this paper.

A two-species population model in which the diffusion of one species depends on the population density of the other species was first proposed by Shigesada, Kawasaki and Teramoto [16] to investigate the habitat segregation phenomena between two competing species. Since their work was published, many mathematicians have studied population models with density-dependent diffusion. However, population models including density-dependent diffusion terms of a fractional type have appeared in recent years; for example, see [8], [19] for cooperative models with Dirichlet boundary conditions; [1], [2], [5], [6] for prey-predator models; [14], [18] for three-species prey-predator models.

The stationary problem associated with $(\mathrm{P})$ is

$$
(\mathrm{SP}) \begin{cases}\Delta\left[\left(1+\frac{\alpha}{\mu+v}\right) u\right]+u(a-u+c v)=0 & \text { in } \Omega, \\ \Delta v+v(-b+d u-v)=0 & \text { in } \Omega, \\ \frac{\partial u}{\partial n}=\frac{\partial v}{\partial n}=0 & \text { on } \partial \Omega .\end{cases}
$$

The main purpose of the present paper is to study the existence of stationary patterns (i.e. positive non-constant solutions) for (SP) with the weak cooperative condition

$$
\frac{a}{b}>\frac{1}{d}>c .
$$

From now on, we always assume (1.1). When $\alpha=0$, the following proposition due to Lou, Nagylaki and $\mathrm{Ni}[9]$ is well known.

Proposition 1.1 (Lou, Nagylaki and $\mathrm{Ni}$ [9]). Let $\alpha=0$. Then every solution of $(\mathrm{P})$ converges to a unique positive constant steady-state

$$
\left(u^{*}, v^{*}\right):=\left(\frac{a-b c}{1-c d}, \frac{a d-b}{1-c d}\right)
$$

uniformly as $t \rightarrow \infty$. 
Proposition 1.1 implies that $\left(u^{*}, v^{*}\right)$ is a unique positive solution of (SP) with $\alpha=0$. Namely, no stationary pattern exists in the linear diffusion case. However, the presence of density-dependent diffusion may enable us to construct stationary patterns of $(\mathrm{SP})$. We focus on $\alpha$ to discuss the existence and the non-existence of stationary patterns for (SP).

We first state the non-existence result of stationary patterns for (SP).

Theorem 1.1. There exists a positive constant $\alpha_{*}=\alpha_{*}(a, b, c, d, \mu)$ such that, if $\alpha \leq \alpha_{*}$, then $\left(u^{*}, v^{*}\right)$ is a unique positive solution of $(\mathrm{SP})$.

Theorem 1.1 means that stationary patterns still do not exist when the effect of density-dependent diffusion is small. However, we can prove the existence of stationary patterns for (SP) if the effect of density-dependent diffusion is large.

Let $0=\lambda_{0}<\lambda_{1}<\lambda_{2}<\cdots$ denote eigenvalues of $-\Delta$ with the homogeneous Neumann boundary condition on $\partial \Omega$ and let $m_{i}$ denote the algebraic multiplicity of $\lambda_{i}$. Then we have the following theorem which is one of our main results.

Theorem 1.2. Assume that $\left\{v^{*}(b-\mu)\right\} /\left(\mu+v^{*}\right) \in\left(\lambda_{l}, \lambda_{l+1}\right)$ for some $l \geq 1$ and that $\sum_{i=1}^{l} m_{i}$ is odd. Then there exists a positive constant $\alpha^{*}=\alpha^{*}(a, b, c, d, \mu)$ such that (SP) has at least one positive non-constant solution for each $\alpha>\alpha^{*}$.

Ecologically, it may be said that as $\alpha$ increases, each individual of species $u$ becomes not only more active, but also more sensitive to the concentration level of species $v$ at the place where the individual is locating. Then Theorems 1.1 and 1.2 indicate that such sensitivity generates stationary patterns. We also remark that the existence of stationary patterns suggests the complexity of the dynamical behavior of $(\mathrm{P})$ for large $\alpha$.

We are also interested in the limiting patterns of (SP) as $\alpha \rightarrow \infty$. Under the restriction $N \leq 3$, we obtain the following limiting system as $\alpha \rightarrow \infty$.

Theorem 1.3. Suppose $N \leq 3$ and $b>\mu$. Let $\left\{\left(u_{i}, v_{i}, \alpha_{i}\right)\right\}_{i=1}^{\infty}$ be any sequence such that $\lim _{i \rightarrow \infty} \alpha_{i}=\infty$ and positive functions $\left(u_{i}, v_{i}\right)$ satisfy (SP) with $\alpha=\alpha_{i}$. Then, by passing to a subsequence if necessary, it holds that

$$
\lim _{i \rightarrow \infty}\left(u_{i}, v_{i}\right)=(\tau(\mu+\bar{v}), \bar{v}) \quad \text { in } C^{1}(\bar{\Omega}) \times C^{1}(\bar{\Omega}),
$$

where $\tau$ is a positive constant satisfying $1<d \tau<b / \mu, \bar{v}$ is a positive function in $\bar{\Omega}$ and $(\tau, \bar{v})$ satisfies

$$
\begin{cases}\Delta \bar{v}+\bar{v}\{-b+d \tau \mu+(d \tau-1) \bar{v}\}=0 & \text { in } \Omega, \\ \frac{\partial \bar{v}}{\partial n}=0 & \text { on } \partial \Omega, \\ \int_{\Omega}(\mu+\bar{v})\{a-\tau \mu+(c-\tau) \bar{v}\} d x=0 . & \end{cases}
$$


We expect that the limiting system (1.2) may give much information on profiles of stationary patterns of (SP) for large $\alpha$. We will study (1.2) in a forthcoming paper.

This paper is organized as follows. In Section 2, we will consider the linearized eigenvalue problem of $(\mathrm{P})$ at $\left(u^{*}, v^{*}\right)$. In Section 3, we will derive different kinds of a priori estimates. These a priori estimates will play a key role in the proofs of Theorems 1.2 and 1.3. In Section 4, we will show Theorem 1.1. In Section 5, we will prove Theorem 1.2 by using the LeraySchauder degree theory (see e.g. [12]). In Section 6, we will show Theorem 1.3.

Throughout the paper, the usual norms of $L^{p}(\Omega)$ for $p \in[1, \infty)$ and $C(\bar{\Omega})$ are defined by

$$
\|\psi\|_{p}:=\left(\int_{\Omega}|\psi(x)|^{p} d x\right)^{1 / p} \quad \text { and } \quad\|\psi\|_{\infty}:=\max _{x \in \bar{\Omega}}|\psi(x)|,
$$

respectively.

\section{Stability of the constant solution $\left(u^{*}, v^{*}\right)$}

In this section, we will analyze the linearized stability of the constant stationary solution $\left(u^{*}, v^{*}\right)$ for $(\mathrm{P})$.

The linearized eigenvalue problem of $(\mathrm{P})$ at $\left(u^{*}, v^{*}\right)$ is given by

$$
\begin{cases}-\left(1+\frac{\alpha}{\mu+v^{*}}\right) \Delta h+\frac{\alpha u^{*}}{\left(\mu+v^{*}\right)^{2}} \Delta k+u^{*} h-c u^{*} k=\eta h & \text { in } \Omega, \\ -\Delta k-d v^{*} h+v^{*} k=\eta k & \text { in } \Omega, \\ \frac{\partial h}{\partial n}=\frac{\partial k}{\partial n}=0 & \text { on } \partial \Omega .\end{cases}
$$

We know that $\left(u^{*}, v^{*}\right)$ is linearly stable when $\alpha=0$. Using the expansions of $h$ and $k$ in terms of eigenfunctions of $-\Delta$, one can see that $\eta$ is an eigenvalue of (2.1) if and only if

$$
\operatorname{det}\left(\begin{array}{cc}
\left(1+\alpha\left(\mu+v^{*}\right)^{-1}\right) \lambda_{i}+u^{*}-\eta & -\alpha u^{*}\left(\mu+v^{*}\right)^{-2} \lambda_{i}-c u^{*} \\
-d v^{*} & \lambda_{i}+v^{*}-\eta
\end{array}\right)=0
$$

for some $i \geq 0$, where $\lambda_{i}$ is an eigenvalue of $-\Delta$ defined in Section 1 . In particular, $\eta=0$ is an eigenvalue of (2.1) if and only if

$$
\frac{\lambda_{i}}{\left(\mu+v^{*}\right)^{2}}\left\{\left(\mu+v^{*}\right)\left(\lambda_{i}+v^{*}\right)-d u^{*} v^{*}\right\} \alpha+\left(\lambda_{i}+u^{*}\right)\left(\lambda_{i}+v^{*}\right)-c d u^{*} v^{*}=0
$$

for some $i \geq 0$. Note that $\left(\lambda_{i}+u^{*}\right)\left(\lambda_{i}+v^{*}\right)-c d u^{*} v^{*}>0$ for all $i \geq 0$ because of (1.1). Thus it is easy to see that the linearized stability of $\left(u^{*}, v^{*}\right)$ changes as 
$\alpha$ increases in $(\mathrm{P})$ if and only if

$$
\begin{aligned}
\left(\mu+v^{*}\right)\left(\lambda_{1}+v^{*}\right)-d u^{*} v^{*} & =\left(\mu+v^{*}\right) \lambda_{1}+v^{*}\left(\mu+v^{*}-d u^{*}\right) \\
& =\left(\mu+v^{*}\right) \lambda_{1}+v^{*}(\mu-b) \\
& <0 .
\end{aligned}
$$

Therefore, $b>\mu$ is necessary for the linearized stability of $\left(u^{*}, v^{*}\right)$ to change (and so we do not discuss the case $b \leq \mu$, especially, $-b \geq 0$ ). This means that the difference in the intrinsic growth rates between two species $u$ and $v$ contributes to creating stationary patterns in (SP).

Remark 2.1. From the viewpoint of the bifurcation theory, the change of the linearized stability of $\left(u^{*}, v^{*}\right)$ implies that positive non-constant solutions of (SP) bifurcate from $\left(u^{*}, v^{*}\right)$ as $\alpha$ increases. In fact, under suitable conditions, we can obtain bifurcation branches of positive non-constant solutions for (SP) by using the local bifurcation theorem [3]. In that case, positive non-constant solutions obtained in Theorem 1.2 probably correspond to one of those bifurcation branches.

Remark 2.2. Proposition 1.1 still holds true even if we replace $\Delta u$ and $\Delta v$ by $v_{1} \Delta u$ and $v_{2} \Delta v$ for any $v_{1}, v_{2}>0$, respectively, whenever $\alpha=0$ in $(\mathrm{P})$; see [9]. Namely, in the linear diffusion case, $(\mathrm{P})$ does not exhibit diffusion-induced instability in Turing's sense (see [17]). However, the argument in this section shows that diffusion-induced instability emerges by the presence of densitydependent diffusion.

\section{Preliminaries and a priori estimates}

\subsection{Preliminaries}

In this section, we will derive some a priori estimates. We first recall the following maximum principle due to Lou and $\mathrm{Ni}[10]$ which will be very useful.

Lemma 3.1. Suppose that $g \in C(\bar{\Omega} \times \boldsymbol{R})$.

(i) If $w \in C^{2}(\Omega) \cap C^{1}(\bar{\Omega})$ satisfies

$$
\Delta w(x)+g(x, w(x)) \geq 0 \quad \text { in } \Omega, \quad \frac{\partial w}{\partial n} \leq 0 \quad \text { on } \partial \Omega,
$$

and $w\left(x_{0}\right)=\max _{\bar{\Omega}} w$, then $g\left(x_{0}, w\left(x_{0}\right)\right) \geq 0$.

(ii) If $w \in C^{2}(\Omega) \cap C^{1}(\bar{\Omega})$ satisfies

$$
\Delta w(x)+g(x, w(x)) \leq 0 \quad \text { in } \Omega, \quad \frac{\partial w}{\partial n} \geq 0 \quad \text { on } \partial \Omega,
$$

and $w\left(x_{0}\right)=\min _{\bar{\Omega}} w$, then $g\left(x_{0}, w\left(x_{0}\right)\right) \leq 0$. 
We also need the following Harnack inequality (see Lin, Ni and Takagi [7], and Lou and Ni [11]).

Lemma 3.2. Suppose that $f \in C(\bar{\Omega})$ with $\max \{N / 2,1\}<p \leq \infty$ and $\|f\|_{p} \leq B$ for some $B>0$. Let $w \in C^{2}(\Omega) \cap C^{1}(\bar{\Omega})$ be a positive solution of $\Delta w+f(x) w=0$ in $\Omega$ subject to the homogeneous Neumann boundary condition. Then there exists a positive constant $C_{\#}=C_{\#}(p, N, \Omega, B)$ such that

$$
\max _{\bar{\Omega}} w \leq C_{\#} \min _{\bar{\Omega}} w .
$$

\subsection{A priori estimates}

In this subsection, we will introduce a semilinear elliptic system (EP), which is equivalent to (SP), and give some a priori estimates for positive solutions of (EP). These a priori estimates will be required for the proof of Theorem 1.2.

We first introduce a new unknown function $U$ by

$$
U=\left(1+\frac{\alpha}{\mu+v}\right) u .
$$

Clearly, there exists a one-to-one correspondence between $(u, v)>0$ and $(U, v)>0$. As far as we discuss positive solutions, (SP) is rewritten in the following equivalent form:

$$
(\mathrm{EP}) \begin{cases}\Delta U+\frac{\mu+v}{\mu+v+\alpha} U\left(a-\frac{\mu+v}{\mu+v+\alpha} U+c v\right)=0 & \text { in } \Omega, \\ \Delta v+v\left(-b+d \frac{\mu+v}{\mu+v+\alpha} U-v\right)=0 & \text { in } \Omega, \\ \frac{\partial U}{\partial n}=\frac{\partial v}{\partial n}=0 & \text { on } \partial \Omega .\end{cases}
$$

Now we can derive the following a priori estimates.

Lemma 3.3. Let $\zeta$ be any fixed positive number. Then there exist two positive constants $C_{*}(\zeta)=C_{*}(\zeta, a, b, c, d, \mu)<C^{*}(\zeta)=C^{*}(\zeta, a, b, c, d, \mu)$ such that, if $\alpha \leq \zeta$, then any positive solution $(U, v)$ of $(\mathrm{EP})$ satisfies

$$
a \leq U(x) \leq C^{*}(\zeta) \quad \text { and } \quad C_{*}(\zeta) \leq v(x) \leq C^{*}(\zeta) \quad \text { for all } x \in \bar{\Omega}
$$

Proof. Let $U\left(x_{0}\right)=\max _{\bar{\Omega}} U$ and $\quad v\left(y_{0}\right)=\max _{\bar{\Omega}} v \quad$ with $\quad x_{0}, y_{0} \in \bar{\Omega}$. Applying Lemma 3.1 to (EP), we have

$$
\max _{\bar{\Omega}} U \leq \frac{\mu+v\left(x_{0}\right)+\alpha}{\mu+v\left(x_{0}\right)}\left(a+c v\left(x_{0}\right)\right)
$$


and

$$
\max _{\bar{\Omega}} v \leq-b+d \frac{\mu+v\left(y_{0}\right)}{\mu+v\left(y_{0}\right)+\alpha} U\left(y_{0}\right) \leq-b+d \max _{\bar{\Omega}} U
$$

Thus

$$
\begin{aligned}
\max _{\bar{\Omega}} U & \leq a+c v\left(x_{0}\right)+\zeta \frac{a+c v\left(x_{0}\right)}{\mu+v\left(x_{0}\right)} \\
& \leq a+c\left(-b+d \max _{\bar{\Omega}} U\right)+\zeta \max \left\{\frac{a}{\mu}, c\right\} .
\end{aligned}
$$

Therefore, we see

$$
\max _{\bar{\Omega}} U \leq \frac{a-b c+\zeta \max \{a / \mu, c\}}{1-c d} .
$$

It follows from (3.2) and (3.3) that

$$
\max _{\bar{\Omega}} v \leq-b+\frac{d(a-b c+\zeta \max \{a / \mu, c\})}{1-c d}=\frac{a d-b+\zeta d \max \{a / \mu, c\}}{1-c d} .
$$

Hence we have obtained the desired upper bound of $(U, v)$.

Let $U\left(z_{0}\right)=\min _{\bar{\Omega}} U$ with some $z_{0} \in \bar{\Omega}$. Using Lemma 3.1 to the first equation of $(\mathrm{EP})$, we get

$$
\min _{\bar{\Omega}} U \geq \frac{\mu+v\left(z_{0}\right)+\alpha}{\mu+v\left(z_{0}\right)}\left(a+c v\left(z_{0}\right)\right) \geq a .
$$

Thus we have obtained the desired lower bound of $U$.

Finally, we derive a lower bound of $v$ by contradiction. Suppose that there exist a positive constant $\zeta_{0}$ and a sequence $\left\{\left(U_{i}, v_{i}, \alpha_{i}\right)\right\}_{i=1}^{\infty}$ such that $\alpha_{i} \leq \zeta_{0}$ for all $i \in N, \lim _{i \rightarrow \infty} \alpha_{i}=\alpha_{\infty}$ for some non-negative constant $\alpha_{\infty}$,

$$
\lim _{i \rightarrow \infty} \min _{\bar{\Omega}} v_{i}=0
$$

and positive functions $\left(U_{i}, v_{i}\right)$ satisfy

$$
\begin{cases}\Delta U_{i}+\frac{\mu+v_{i}}{\mu+v_{i}+\alpha_{i}} U_{i}\left(a-\frac{\mu+v_{i}}{\mu+v_{i}+\alpha_{i}} U_{i}+c v_{i}\right)=0 & \text { in } \Omega, \\ \Delta v_{i}+v_{i}\left(-b+d \frac{\mu+v_{i}}{\mu+v_{i}+\alpha_{i}} U_{i}-v_{i}\right)=0 & \text { in } \Omega, \\ \frac{\partial U_{i}}{\partial n}=\frac{\partial v_{i}}{\partial n}=0 & \text { on } \partial \Omega .\end{cases}
$$


By using the regularity theory for elliptic equations (see e.g. [4]) to the second equation of (3.7), it follows from (3.3) and (3.4) that

$$
\left\|v_{i}\right\|_{W^{2, p}(\Omega)} \leq C\left(\zeta_{0}\right)
$$

with some positive constant $C\left(\zeta_{0}\right)=C\left(\zeta_{0}, a, b, c, d, \mu\right)$ independent of $i$. If $p>N$, then Sobolev's embedding theorem implies $\left\{v_{i}\right\}_{i=1}^{\infty}$ is compact in $C^{1}(\bar{\Omega})$. Consequently, there exists a subsequence, which is still denoted by $\left\{v_{i}\right\}_{i=1}^{\infty}$, such that

$$
\lim _{i \rightarrow \infty} v_{i}=v_{\infty} \quad \text { in } C^{1}(\bar{\Omega})
$$

with some non-negative function $v_{\infty} \in C^{1}(\bar{\Omega})$. Similarly, there exists a nonnegative function $U_{\infty} \in C^{1}(\bar{\Omega})$ such that

$$
\lim _{i \rightarrow \infty} U_{i}=U_{\infty} \quad \text { in } C^{1}(\bar{\Omega}) .
$$

Therefore, $v_{\infty}$ satisfies

$$
\Delta v_{\infty}+v_{\infty}\left(-b+d \frac{\mu+v_{\infty}}{\mu+v_{\infty}+\alpha_{\infty}} U_{\infty}-v_{\infty}\right)=0 \quad \text { in } \Omega, \quad \frac{\partial v_{\infty}}{\partial n}=0 \quad \text { on } \partial \Omega
$$

in the weak sense. By standard elliptic regularity theory we have $v_{\infty} \in C^{2}(\bar{\Omega})$ and thus $v_{\infty}$ is a classical solution of the above equation. Then it follows from (3.6), (3.8) and the strong maximum principle that $v_{\infty} \equiv 0$ in $\bar{\Omega}$. We can easily see from the above argument that $U_{\infty}$ satisfies

$$
\Delta U_{\infty}+\frac{\mu}{\mu+\alpha_{\infty}} U_{\infty}\left(a-\frac{\mu}{\mu+\alpha_{\infty}} U_{\infty}\right)=0 \quad \text { in } \Omega, \quad \frac{\partial U_{\infty}}{\partial n}=0 \quad \text { on } \partial \Omega
$$

in the classical sense. Then by the strong maximum principle and Lemma 3.1, either $U_{\infty} \equiv a\left(\mu+\alpha_{\infty}\right) / \mu$ or $U_{\infty} \equiv 0$ in $\bar{\Omega}$. Combining (3.5) and (3.9), we can conclude $U_{\infty} \equiv a\left(\mu+\alpha_{\infty}\right) / \mu$ in $\bar{\Omega}$. Hence

$$
\lim _{i \rightarrow \infty}\left(-b+d \frac{\mu+v_{i}}{\mu+v_{i}+\alpha_{i}} U_{i}-v_{i}\right)=a d-b>0 \quad \text { uniformly in } \Omega
$$

by (1.1) and this means

$$
v_{i}\left(-b+d \frac{\mu+v_{i}}{\mu+v_{i}+\alpha_{i}} U_{i}-v_{i}\right)>0 \quad \text { in } \Omega
$$

for sufficiently large $i \in N$ because $v_{i}>0$ in $\Omega$. On the other hand, from the second equation of (3.7), we have

$$
\int_{\Omega} v_{i}\left(-b+d \frac{\mu+v_{i}}{\mu+v_{i}+\alpha_{i}} U_{i}-v_{i}\right) d x=-\int_{\Omega} \Delta v_{i} d x=-\int_{\partial \Omega} \frac{\partial v_{i}}{\partial n} d \sigma=0
$$

for all $i \in N$. This is a contradiction; thus our proof is complete. 


\subsection{A priori estimates independent of $a$}

In this subsection, we will give some a priori estimates independent of $\alpha$ under the restriction $N \leq 3$. These a priori estimates will play an important role in the proof of Theorem 1.3.

Lemma 3.4. Suppose that $N \leq 3$. Then there exists a positive constant $C_{0}=C_{0}(a, b, c, d, \mu)$ independent of $\alpha$ such that any positive solution $(u, v)$ of (SP) satisfies

$$
\|u\|_{\infty} \leq C_{0} \quad \text { and } \quad\|v\|_{\infty} \leq C_{0} .
$$

Proof. Integrating the second equation of (SP) over $\Omega$ we see

$$
\int_{\Omega} v^{2} d x=-b \int_{\Omega} v d x+d \int_{\Omega} u v d x \leq d\|u\|_{2}\|v\|_{2},
$$

where we have used Schwarz's inequality. Thus

$$
\|v\|_{2} \leq d\|u\|_{2} \text {. }
$$

Similarly,

$$
\int_{\Omega} u^{2} d x=a \int_{\Omega} u d x+c \int_{\Omega} u v d x \leq a|\Omega|^{1 / 2}\|u\|_{2}+c\|u\|_{2}\|v\|_{2},
$$

where $|\Omega|$ denotes the measure of $\Omega$. Then it follows from (3.10) that

$$
\|u\|_{2} \leq a|\Omega|^{1 / 2}+c d\|u\|_{2} .
$$

Combining (3.10) and (3.11), we have

$$
\|u\|_{2} \leq \frac{a}{1-c d}|\Omega|^{1 / 2} \quad \text { and } \quad\|v\|_{2} \leq \frac{a d}{1-c d}|\Omega|^{1 / 2},
$$

which, in particular, give

$$
\min _{\bar{\Omega}} u \leq \frac{a}{1-c d} \quad \text { and } \quad \min _{\bar{\Omega}} v \leq \frac{a d}{1-c d} .
$$

Making use of (3.12), (3.13) and Lemma 3.2 with $p=2$ to the second equation of (SP), we obtain

$$
\max _{\bar{\Omega}} v \leq C_{1} \min _{\bar{\Omega}} v \leq C_{1} \frac{a d}{1-c d}=: C_{2}
$$

for some positive constant $C_{1}=C_{1}(a, b, c, d)$ independent of $\alpha$, where we have used the assumption $N \leq 3$. Thus we have obtained an upper bound of $v$. 
We next derive an upper bound of $u$. Let $U$ be the function defined by (3.1). By (3.12),

$$
\left\|\frac{\mu+v}{\mu+v+\alpha}\left(a-\frac{\mu+v}{\mu+v+\alpha} U+c v\right)\right\|_{2} \leq a+\|u\|_{2}+c\|v\|_{2} \leq C_{3}
$$

for some positive constant $C_{3}=C_{3}(a, c, d)$ independent of $\alpha$. Then applying Lemma 3.2 with $p=2$ to the first equation of (EP), we have

$$
\begin{aligned}
\left(1+\frac{\alpha}{\mu+C_{2}}\right) \max _{\bar{\Omega}} u & \leq \max _{\bar{\Omega}} U \\
& \leq C_{4} \min _{\bar{\Omega}} U \\
& \leq C_{4}\left(1+\frac{\alpha}{\mu}\right) \min _{\bar{\Omega}} u \\
& \leq C_{4}\left(1+\frac{\alpha}{\mu}\right) \frac{a}{1-c d}
\end{aligned}
$$

for some positive constant $C_{4}=C_{4}(a, c, d)$ independent of $\alpha$, where we have used (3.13) and (3.14). Hence we obtain

$$
\max _{\bar{\Omega}} u \leq \frac{C_{4}\left(1+\alpha \mu^{-1}\right)}{1+\alpha\left(\mu+C_{2}\right)^{-1}} \cdot \frac{a}{1-c d} \leq \frac{C_{4}\left(\mu+C_{2}\right)}{\mu} \cdot \frac{a}{1-c d} .
$$

Therefore we get the assertion.

\section{Proof of Theorem 1.1}

In this section, we will prove Theorem 1.1 by using Lemma 3.1.

Set

$$
\begin{aligned}
& \Gamma_{1}:=\left\{(U, v) \in \boldsymbol{R}_{+}^{2} \mid f_{1}(U, v):=a-\frac{\mu+v}{\mu+v+\alpha} U+c v=0\right\}, \\
& \Gamma_{2}:=\left\{(U, v) \in \boldsymbol{R}_{+}^{2} \mid f_{2}(U, v):=-b+d \frac{\mu+v}{\mu+v+\alpha} U-v=0\right\},
\end{aligned}
$$

where $\boldsymbol{R}_{+}^{2}:=\left\{(U, v) \in \boldsymbol{R}^{2} \mid U \geq 0, v \geq 0\right\}$. Moreover, in view of (3.1), we set

$$
U_{\alpha}^{*}=\left(1+\frac{\alpha}{\mu+v^{*}}\right) u^{*}
$$

We first show the following lemma. 
Lemma 4.1. There exists a positive constant $\alpha_{*}=\alpha_{*}(a, b, c, d, \mu)$ such that, if $\alpha \leq \alpha_{*}$, then

$$
\begin{aligned}
& I:=\left\{(U, v) \in \boldsymbol{R}_{+}^{2} \mid f_{1}(U, v) \geq 0, f_{2}(U, v) \geq 0\right\} \subset\left[0, U_{\alpha}^{*}\right] \times\left[0, v^{*}\right], \\
& I I:=\left\{(U, v) \in \boldsymbol{R}_{+}^{2} \mid f_{1}(U, v) \leq 0, f_{2}(U, v) \leq 0\right\} \subset\left[U_{\alpha}^{*}, \infty\right) \times\left[v^{*}, \infty\right) .
\end{aligned}
$$

Proof. Set

$$
g_{1}(v):=\frac{(a+c v)(\mu+v+\alpha)}{\mu+v}, \quad g_{2}(v):=\frac{(b+v)(\mu+v+\alpha)}{d(\mu+v)} .
$$

Then we find $g_{1}\left(v^{*}\right)=g_{2}\left(v^{*}\right)=U_{\alpha}^{*}$,

$$
\Gamma_{1}=\left\{\left(g_{1}(v), v\right) \mid v \geq 0\right\}, \quad \Gamma_{2}=\left\{\left(g_{2}(v), v\right) \mid v \geq 0\right\} .
$$

By (1.1),

$$
g_{1}(0)=\frac{a(\mu+\alpha)}{\mu}>\frac{b(\mu+\alpha)}{d \mu}=g_{2}(0),
$$

and

$$
g_{1}^{\prime}(v)=c-\frac{a-c \mu}{(\mu+v)^{2}} \alpha<\frac{1}{d}-\frac{b-\mu}{d(\mu+v)^{2}} \alpha=g_{2}^{\prime}(v)
$$

for any $v \geq 0$. So there exists a positive constant $\alpha_{*}=\alpha_{*}(a, b, c, d, \mu)$ such that, if $\alpha \leq \alpha_{*}$, then $g_{2}^{\prime}(v)>g_{1}^{\prime}(v)>0$ for any $v \geq 0$. Then we can easily obtain the conclusion.

Figure 1 illustrates ideas of the proofs of Lemma 4.1 and Theorem 1.1. We are now in a position to prove Theorem 1.1. Owing to (3.1), it suffices to consider (EP) instead of (SP).

Proof of Theorem 1.1. Suppose that $(U, v)$ is any positive solution of (EP) with $\alpha \leq \alpha_{*}$, where $\alpha_{*}$ is a constant given in Lemma 4.1. Let $U\left(x_{1}\right)=\max _{\bar{\Omega}} U$, $U\left(x_{2}\right)=\min _{\bar{\Omega}} U, v\left(x_{3}\right)=\max _{\bar{\Omega}} v$ and $v\left(x_{4}\right)=\min _{\bar{\Omega}} v$ with $x_{i} \in \bar{\Omega}(i=1,2,3,4)$. Then by Lemma 3.1, we have

$$
\begin{aligned}
& f_{1}\left(\max _{\bar{\Omega}} U, v\left(x_{1}\right)\right) \geq 0, \\
& f_{1}\left(\min _{\bar{\Omega}} U, v\left(x_{2}\right)\right) \leq 0,
\end{aligned}
$$




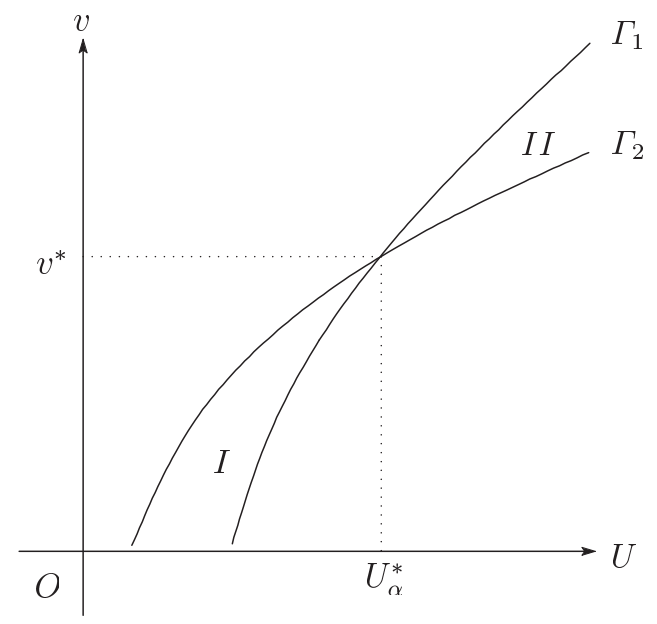

Fig. 1

and since $f_{2}(U, v)$ is monotone increasing with respect to $U$ for any fixed $v \geq 0$, we also have

$$
\begin{aligned}
& f_{2}\left(\max _{\bar{\Omega}} U, \max _{\bar{\Omega}} v\right) \geq f_{2}\left(U\left(x_{3}\right), \max _{\bar{\Omega}} v\right) \geq 0, \\
& f_{2}\left(\min _{\bar{\Omega}} U, \min _{\bar{\Omega}} v\right) \leq f_{2}\left(U\left(x_{4}\right), \min _{\bar{\Omega}} v\right) \leq 0 .
\end{aligned}
$$

On account of (4.2) and $\max _{\bar{\Omega}} v \geq v\left(x_{1}\right)$, we can easily verify

$$
f_{1}\left(\max _{\bar{\Omega}} U, \max _{\bar{\Omega}} v\right) \geq 0 .
$$

Similarly,

$$
f_{1}\left(\min _{\bar{\Omega}} U, \min _{\bar{\Omega}} v\right) \leq 0
$$

Combining (4.3), (4.4) and Lemma 4.1, we have

$$
\left(\max _{\bar{\Omega}} U, \max _{\bar{\Omega}} v\right) \in\left[0, U_{\alpha}^{*}\right] \times\left[0, v^{*}\right] .
$$

Similarly,

$$
\left(\min _{\bar{\Omega}} U, \min _{\bar{\Omega}} v\right) \in\left[U_{\alpha}^{*}, \infty\right) \times\left[v^{*}, \infty\right) .
$$

Therefore, $U \equiv U_{\alpha}^{*}$ and $v \equiv v^{*}$ in $\bar{\Omega}$. This completes the proof. 


\section{Proof of Theorem 1.2}

In this section, we will prove Theorem 1.2 by using a priori estimates (Lemma 3.3) and Leray-Schauder degree theory (see e.g. [12]). Owing to (3.1), it is sufficient to show the existence of positive non-constant solutions of (EP).

Set $X=C(\bar{\Omega}) \times C(\bar{\Omega})$. For each $\alpha \geq 0$, define an operator $F_{\alpha}$ by

$$
F_{\alpha}\left(\begin{array}{l}
U \\
v
\end{array}\right)=\left(\begin{array}{c}
(-\Delta+I)^{-1}\left[U+(\mu+v)(\mu+v+\alpha)^{-1}\right. \\
\left.\times U\left(a-(\mu+v)(\mu+v+\alpha)^{-1} U+c v\right)\right] \\
(-\Delta+I)^{-1}\left[v+v\left(-b+d(\mu+v)(\mu+v+\alpha)^{-1} U-v\right)\right]
\end{array}\right)
$$

where $I$ is the identity map from $C(\bar{\Omega})$ into itself, and $(-\Delta+I)^{-1}$ is the inverse operator of $-\Delta+I$ subject to the homogeneous Neumann boundary condition on $\partial \Omega$. It is easy to see that $F_{\alpha}: X \rightarrow X$ is well-defined, and that by elliptic regularity theory and Sobolev's embedding theorem, $F_{\alpha}$ is a continuous and compact operator for each $\alpha \geq 0$. From these observations, one can define the Leray-Schauder degree of $I-F_{\alpha}$ at 0 in a suitable open set. Furthermore, $(U, v)$ is a positive solution of $\left(I-F_{\alpha}\right)(U, v)=0$ if and only if $(U, v)$ is a positive solution of (EP).

Note that $\left(u^{*}, v^{*}\right)$ is a zero point of $I-F_{0}$. We first calculate the index of $I-F_{0}$ at $\left(u^{*}, v^{*}\right)$, denoted by $\operatorname{index}\left(I-F_{0},\left(u^{*}, v^{*}\right)\right)$.

Lemma 5.1. It holds that $\operatorname{index}\left(I-F_{0},\left(u^{*}, v^{*}\right)\right)=1$.

Proof. From (5.1), $F_{0}$ is given by

$$
F_{0}\left(\begin{array}{c}
U \\
v
\end{array}\right)=\left(\begin{array}{c}
(-\Delta+I)^{-1}[U+U(a-U+c v)] \\
(-\Delta+I)^{-1}[v+v(-b+d U-v)]
\end{array}\right) .
$$

Some elementary calculations show

$$
D F_{0}\left(u^{*}, v^{*}\right)\left(\begin{array}{l}
h \\
k
\end{array}\right)=\left(\begin{array}{c}
(-\Delta+I)^{-1}\left[\left(1-u^{*}\right) h+c u^{*} k\right] \\
(-\Delta+I)^{-1}\left[d v^{*} h+\left(1-v^{*}\right) k\right]
\end{array}\right),
$$

where $D F_{0}\left(u^{*}, v^{*}\right)$ denotes the Fréchet derivative of $F_{0}$ at $\left(u^{*}, v^{*}\right)$. Since $\left(u^{*}, v^{*}\right)$ is a unique positive solution of (SP) with $\alpha=0,\left(u^{*}, v^{*}\right)$ is an isolated zero point of $I-F_{0}$. Thus by the standard Leray-Schauder degree theory,

$$
\operatorname{index}\left(I-F_{0},\left(u^{*}, v^{*}\right)\right)=(-1)^{\sigma},
$$

where $\sigma$ is the number of negative eigenvalues of $I-D F_{0}\left(u^{*}, v^{*}\right)$ (counting algebraic multiplicity). Note that $\eta$ is an eigenvalue of $I-D F_{0}\left(u^{*}, v^{*}\right)$ if and 
only if there exists some $(h, k) \neq(0,0)$ such that $\left(I-D F_{0}\left(u^{*}, v^{*}\right)\right)(h, k)=$ $\eta(h, k)$, i.e.,

$$
\begin{cases}-(1-\eta) \Delta h+\left(-\eta+u^{*}\right) h-c u^{*} k=0 & \text { in } \Omega, \\ -(1-\eta) \Delta k+\left(-\eta+v^{*}\right) k-d v^{*} h=0 & \text { in } \Omega, \\ \frac{\partial h}{\partial n}=\frac{\partial k}{\partial n}=0 & \text { on } \partial \Omega .\end{cases}
$$

Set

$$
M_{i}(\eta)=\left(\begin{array}{cc}
(1-\eta) \lambda_{i}-\eta+u^{*} & -c u^{*} \\
-d v^{*} & (1-\eta) \lambda_{i}-\eta+v^{*}
\end{array}\right)
$$

where $\lambda_{i}$ is an eigenvalue of $-\Delta$ defined in Section 1. Observe that $\eta$ is an eigenvalue of $I-D F_{0}\left(u^{*}, v^{*}\right)$ if and only if $\operatorname{det} M_{i}(\eta)=0$ for some $i \geq 0$. We find from (1.1) that

$$
\begin{aligned}
\operatorname{det} M_{i}(\eta) & =\left\{(1-\eta) \lambda_{i}-\eta+u^{*}\right\}\left\{(1-\eta) \lambda_{i}-\eta+v^{*}\right\}-c d u^{*} v^{*} \\
& =\left\{(1-\eta) \lambda_{i}-\eta\right\}^{2}+\left\{(1-\eta) \lambda_{i}-\eta\right\}\left(u^{*}+v^{*}\right)+(1-c d) u^{*} v^{*} \\
& >0
\end{aligned}
$$

for all $\eta<0$ and all $i \geq 0$. This fact implies that $I-D F_{0}\left(u^{*}, v^{*}\right)$ has no negative eigenvalue. Therefore, $\sigma=0$ and $\operatorname{index}\left(I-F_{0},\left(u^{*}, v^{*}\right)\right)=1$.

Note that $\left(U_{\alpha}^{*}, v^{*}\right)$ is a zero point of $I-F_{\alpha}$, where $U_{\alpha}^{*}$ is a constant defined by (4.1). We next calculate $\operatorname{index}\left(I-F_{\alpha},\left(U_{\alpha}^{*}, v^{*}\right)\right)$ for sufficiently large $\alpha$. Setting

$$
\gamma^{*}:=\frac{v^{*}(b-\mu)}{\mu+v^{*}}=v^{*}\left(\frac{d u^{*}}{\mu+v^{*}}-1\right)
$$

we have the following lemma.

Lemma 5.2. Suppose that

$$
\gamma^{*} \in\left(\lambda_{l}, \lambda_{l+1}\right)
$$

for some $l \geq 1$. There exists a positive constant $\alpha^{*}=\alpha^{*}(a, b, c, d, \mu)$ such that if $\alpha>\alpha^{*}$, then

$$
\operatorname{index}\left(I-F_{\alpha},\left(U_{\alpha}^{*}, v^{*}\right)\right)=(-1)^{\sum_{i=1}^{l} m_{i}}
$$

where $m_{i}$ denotes the algebraic multiplicity of $\lambda_{i}$ defined in Section 1. 
Proof. We differentiate (5.1) to obtain

$$
\begin{aligned}
& D F_{\alpha}\left(U_{\alpha}^{*}, v^{*}\right)\left(\begin{array}{l}
h \\
k
\end{array}\right) \\
& =\left(\begin{array}{c}
(-\Delta+I)^{-1}\left[\left\{1-u^{*}\left(\mu+v^{*}\right)\left(\mu+v^{*}+\alpha\right)^{-1}\right\} h\right. \\
\left.+u^{*}\left\{-\alpha u^{*}\left(\mu+v^{*}+\alpha\right)^{-1}\left(\mu+v^{*}\right)^{-1}+c\right\} k\right] \\
(-\Delta+I)^{-1}\left[\begin{array}{c}
d v^{*}\left(\mu+v^{*}\right)\left(\mu+v^{*}+\alpha\right)^{-1} h \\
\left.+\left\{1+v^{*}\left(\alpha d u^{*}\left(\mu+v^{*}+\alpha\right)^{-1}\left(\mu+v^{*}\right)^{-1}-1\right)\right\} k\right]
\end{array}\right) .
\end{array}\right.
\end{aligned}
$$

If $\left(U_{\alpha}^{*}, v^{*}\right)$ is an isolated zero point of $I-F_{\alpha}$, then

$$
\operatorname{index}\left(I-F_{\alpha},\left(U_{\alpha}^{*}, v^{*}\right)\right)=(-1)^{\sigma},
$$

where $\sigma$ is the number of negative eigenvalues of $I-D F_{\alpha}\left(U_{\alpha}^{*}, v^{*}\right)$ (counting algebraic multiplicity). By the same argument as the proof of Lemma 5.1, $\eta$ is an eigenvalue of $I-D F_{\alpha}\left(U_{\alpha}^{*}, v^{*}\right)$ if and only if det $N_{i}^{\alpha}(\eta)=0$ for some $i \geq 0$, where

$$
\begin{aligned}
& N_{i}^{\alpha}(\eta) \\
& =\left(\begin{array}{ll}
(1-\eta) \lambda_{i}-\eta+u^{*}\left(\mu+v^{*}\right) & u^{*}\left\{\alpha u^{*}\left(\mu+v^{*}+\alpha\right)^{-1}\left(\mu+v^{*}\right)^{-1}-c\right\} \\
\times\left(\mu+v^{*}+\alpha\right)^{-1} & \\
-d v^{*}\left(\mu+v^{*}\right)\left(\mu+v^{*}+\alpha\right)^{-1} & (1-\eta) \lambda_{i}-\eta-v^{*}\left\{\alpha d u^{*}\left(\mu+v^{*}+\alpha\right)^{-1}\right. \\
& \left.\times\left(\mu+v^{*}\right)^{-1}-1\right\}
\end{array}\right) .
\end{aligned}
$$

Moreover,

$$
\sigma=\sum_{i \geq 0} \sum_{\eta_{i} \in \Lambda_{i}} \xi_{\eta_{i}},
$$

where $\Lambda_{i}$ is the set of negative roots of $\operatorname{det} N_{i}^{\alpha}(\eta)=0, \xi_{\eta_{i}}=\eta_{i}^{*} m_{i}$ and $\eta_{i}^{*}$ is the multiplicity of $\eta_{i}$ as a root of $\operatorname{det} N_{i}^{\alpha}(\eta)=0$. We remark that for any fixed $\alpha$ and $i$, det $N_{i}^{\alpha}(\eta)$ forms a quadratic function with respect to $\eta$ expressed by

$$
\begin{aligned}
\operatorname{det} N_{i}^{\alpha}(\eta)= & \left\{-\left(1+\lambda_{i}\right) \eta+\lambda_{i}+\frac{u^{*}\left(\mu+v^{*}\right)}{\mu+v^{*}+\alpha}\right\} \\
& \times\left[-\left(1+\lambda_{i}\right) \eta+\lambda_{i}-v^{*}\left\{\frac{\alpha d u^{*}}{\left(\mu+v^{*}+\alpha\right)\left(\mu+v^{*}\right)}-1\right\}\right] \\
& +\frac{d u^{*} v^{*}\left(\mu+v^{*}\right)}{\mu+v^{*}+\alpha}\left\{\frac{\alpha u^{*}}{\left(\mu+v^{*}+\alpha\right)\left(\mu+v^{*}\right)}-c\right\}
\end{aligned}
$$


Furthermore, $\operatorname{det} N_{i}^{\alpha}(\eta)$ approaches a limiting quadratic function as $\alpha \rightarrow \infty$ :

$$
\lim _{\alpha \rightarrow \infty} \operatorname{det} N_{i}^{\alpha}(\eta)=\left\{\left(1+\lambda_{i}\right) \eta-\lambda_{i}\right\}\left\{\left(1+\lambda_{i}\right) \eta-\left(\lambda_{i}-\gamma^{*}\right)\right\} .
$$

We will divide the derivation of $\sigma$ into three cases for $i$ : $i=0,1 \leq i \leq l$, and $i \geq l+1$.

Case 1. $\quad i=0$. Since $\lambda_{0}=0,(5.3)$ and (5.4) yield

$$
\operatorname{det} N_{0}^{\alpha}(0)=\frac{(1-c d)\left(\mu+v^{*}\right) u^{*} v^{*}}{\mu+v^{*}+\alpha}>0
$$

for all $\alpha>0$ by $(1.1)$ and

$$
\lim _{\alpha \rightarrow \infty} \operatorname{det} N_{0}^{\alpha}(\eta)=\eta\left(\eta+\gamma^{*}\right) .
$$

Since $\gamma^{*}>0$ by (5.2), there exists a positive constant $\alpha_{0}=\alpha_{0}(a, b, c, d, \mu)$ such that, if $\alpha>\alpha_{0}$, then the equation $\operatorname{det} N_{0}^{\alpha}(\eta)=0$ has exactly two negative roots.

Case 2. $1 \leq i \leq l$. By (5.2), $\lambda_{i}-\gamma^{*}<0$ for all $i \in\{1,2, \ldots, l\}$. So it follows from (5.4) that for each $i \in\{1,2, \ldots, l\}$, there exists a positive constant $\alpha_{i}=\alpha_{i}(a, b, c, d, \mu)$ such that, if $\alpha>\alpha_{i}$, then the equation $\operatorname{det} N_{i}^{\alpha}(\eta)=0$ has one positive root and one negative root.

Case 3. $i \geq l+1$. We see from (5.2) that

$$
\begin{aligned}
\lim _{\alpha \rightarrow \infty}\left[\lambda_{i}-v^{*}\left\{\frac{\alpha d u^{*}}{\left(\mu+v^{*}+\alpha\right)\left(\mu+v^{*}\right)}-1\right\}\right] & =\lambda_{i}-v^{*}\left(\frac{d u^{*}}{\mu+v^{*}}-1\right) \\
& =\lambda_{i}-\gamma^{*}>0
\end{aligned}
$$

and that

$$
\lambda_{i}-v^{*}\left\{\frac{\alpha d u^{*}}{\left(\mu+v^{*}+\alpha\right)\left(\mu+v^{*}\right)}-1\right\}=\lambda_{i}-v^{*}\left\{\frac{d u^{*}}{\left(\left(\mu+v^{*}\right) \alpha^{-1}+1\right)\left(\mu+v^{*}\right)}-1\right\}
$$

is monotone decreasing with respect to $\alpha>0$. Hence in (5.3),

$$
\lambda_{i}-v^{*}\left\{\frac{\alpha d u^{*}}{\left(\mu+v^{*}+\alpha\right)\left(\mu+v^{*}\right)}-1\right\}>0
$$

for all $\alpha>0$ and all $i \geq l+1$. Consequently, we obtain

$$
\operatorname{det} N_{i}^{\alpha}(\eta) \geq \operatorname{det} N_{l+1}^{\alpha}(0)
$$

for all $\alpha>0$, all $i \geq l+1$ and all $\eta<0$, and

$$
\lim _{\alpha \rightarrow \infty} \operatorname{det} N_{l+1}^{\alpha}(0)=\lambda_{l+1}\left(\lambda_{l+1}-\gamma^{*}\right)>0 .
$$


Therefore, there exists a positive constant $\alpha_{l+1}=\alpha_{l+1}(a, b, c, d, \mu)$ such that, if $\alpha>\alpha_{l+1}$, then the equation $\operatorname{det} N_{i}^{\alpha}(\eta)=0$ has no negative root for any $i \geq l+1$.

Define $\alpha^{*}:=\max _{0 \leq i \leq l+1} \alpha_{i}$. Combining the results in cases $1-3$, we see that, if $\alpha>\alpha^{*}$, then 0 is not an eigenvalue of $I-D F_{\alpha}\left(U_{\alpha}^{*}, v^{*}\right)$. This implies that $I-D F_{\alpha}\left(U_{\alpha}^{*}, v^{*}\right)$ is a homeomorphism from $X$ into itself and hence $\left(U_{\alpha}^{*}, v^{*}\right)$ is an isolated zero point of $I-F_{\alpha}$. Therefore combining the results in cases $1-3$ again, we have $\sigma=2 m_{0}+\sum_{i=1}^{l} m_{i}$. Thus

$$
(-1)^{\sigma}=(-1)^{2 m_{0}+\sum_{i=1}^{l} m_{i}}=(-1)^{\sum_{i=1}^{l} m_{i}}
$$

which completes the proof.

By virtue of Lemmas 5.1 and 5.2, we are ready to prove Theorem 1.2. In the proof of Theorem 1.2, we represent $(\mathrm{EP})$ as $(\mathrm{EP})_{\alpha}$ to indicate the dependence on $\alpha$.

Proof of Theorem 1.2. Fix any $\alpha>\alpha^{*}$, where $\alpha^{*}$ is a constant given in Lemma 5.2. It follows from Lemma 3.3 that there exist two positive constants $C_{*}(\alpha)=C_{*}(\alpha, a, b, c, d, \mu)<C^{*}(\alpha)=C^{*}(\alpha, a, b, c, d, \mu)$ such that

$$
a \leq U(x) \leq C^{*}(\alpha) \quad \text { and } \quad C_{*}(\alpha) \leq v(x) \leq C^{*}(\alpha) \quad \text { for all } x \in \bar{\Omega} .
$$

We define

$$
S=\left\{(U, v) \in X \mid \frac{a}{2} \leq U \leq 2 C^{*}(\alpha), \frac{C_{*}(\alpha)}{2} \leq v \leq 2 C^{*}(\alpha) \text { in } \bar{\Omega}\right\} ;
$$

so that $I-F_{v}$ has no zero point on the boundary of $S$ for any $v \in[0, \alpha]$. Note that $I-F_{0}$ has a unique zero point $\left(u^{*}, v^{*}\right)$ in $S$. On account of the homotopy invariance of the Leray-Schauder degree and Lemma 5.1, we have

$$
\operatorname{deg}\left(I-F_{\alpha}, S, 0\right)=\operatorname{deg}\left(I-F_{0}, S, 0\right)=\operatorname{index}\left(I-F_{0},\left(u^{*}, v^{*}\right)\right)=1 .
$$

Suppose that $(\mathrm{EP})_{\alpha}$ has no positive non-constant solution, i.e. $I-F_{\alpha}$ has a unique zero point $\left(U_{\alpha}^{*}, v^{*}\right)$ in $S$. Then from the assumption $\sum_{i=1}^{l} m_{i}$ being odd and Lemma 5.2, it follows that

$$
\operatorname{deg}\left(I-F_{\alpha}, S, 0\right)=\operatorname{index}\left(I-F_{\alpha},\left(U_{\alpha}^{*}, v^{*}\right)\right)=(-1)^{\sum_{i=1}^{l} m_{i}}=-1,
$$

which contradicts (5.5). Thus we complete the proof.

\section{Proof of Theorem 1.3}

In this section, we will prove Theorem 1.3 by using a priori estimates (Lemma 3.4). 
Proof of Theorem 1.3. Let $\left\{\left(u_{i}, v_{i}, \alpha_{i}\right)\right\}_{i=1}^{\infty}$ be any sequence satisfying the conditions in Theorem 1.3. Set

$$
\psi_{i}=\left(\frac{1}{\alpha_{i}}+\frac{1}{\mu+v_{i}}\right) u_{i} .
$$

Note that positive functions $\left(\psi_{i}, v_{i}\right)$ satisfy

$$
\begin{cases}\Delta \psi_{i}+\frac{u_{i}\left(a-u_{i}+c v_{i}\right)}{\alpha_{i}}=0 & \text { in } \Omega, \\ \Delta v_{i}+v_{i}\left(-b+d u_{i}-v_{i}\right)=0 & \text { in } \Omega, \\ \frac{\partial \psi_{i}}{\partial n}=\frac{\partial v_{i}}{\partial n}=0 & \text { on } \partial \Omega,\end{cases}
$$

and that $\left\{\psi_{i}\right\}_{i=1}^{\infty}$ is bounded independently of $i$ by Lemma 3.4. Then by the same compactness argument as the proof of (3.8), there exists a subsequence, which is still denoted by $\left\{\psi_{i}\right\}_{i=1}^{\infty}$, such that

$$
\lim _{i \rightarrow \infty} \psi_{i}=\tau \quad \text { in } C^{1}(\bar{\Omega})
$$

for a non-negative function $\tau \in C^{1}(\bar{\Omega})$. Similarly, we see

$$
\lim _{i \rightarrow \infty} v_{i}=\bar{v} \quad \text { in } C^{1}(\bar{\Omega})
$$

for a non-negative function $\bar{v} \in C^{1}(\bar{\Omega})$. Therefore, we obtain

$$
\lim _{i \rightarrow \infty} u_{i}=\lim _{i \rightarrow \infty} \frac{\psi_{i}}{1 / \alpha_{i}+1 /\left(\mu+v_{i}\right)}=\tau(\mu+\bar{v}) \quad \text { in } C^{1}(\bar{\Omega}) .
$$

We will show that $\tau$ is a positive constant. Observe that $\tau$ satisfies

$$
\Delta \tau=0 \quad \text { in } \Omega, \quad \frac{\partial \tau}{\partial n}=0 \quad \text { on } \partial \Omega
$$

in the weak sense. A standard elliptic regularity theory ensures $\tau \in C^{2}(\bar{\Omega})$; so that $\tau$ must be a non-negative constant. Let $v_{i}\left(x_{i}\right)=\max _{\bar{\Omega}} v_{i}$ with some $x_{i} \in \bar{\Omega}$. It follows from Lemma 3.1 that

$$
u_{i}\left(x_{i}\right) \geq \frac{b+v_{i}\left(x_{i}\right)}{d}>\frac{b}{d}(>0)
$$

for all $i \in N$. This fact, together with (6.2), yields $\tau>0$.

We next prove that $\bar{v}>0$ in $\bar{\Omega}$ and that $(\tau, \bar{v})$ satisfies (1.2). Note that $\bar{v}$ satisfies

$$
\Delta \bar{v}+\bar{v}\{-b+d \tau \mu+(d \tau-1) \bar{v}\}=0 \quad \text { in } \Omega, \quad \frac{\partial \bar{v}}{\partial n}=0 \quad \text { on } \partial \Omega
$$


in the weak sense. In the standard manner, one can see that $\bar{v} \in C^{2}(\bar{\Omega})$ and $\bar{v}$ is a classical nonnegative solution of (6.3). It follows from the strong maximum principle that either $\bar{v} \equiv 0$ or $\bar{v}>0$ in $\bar{\Omega}$. We show $\bar{v}>0$ in $\bar{\Omega}$ by contradiction. Suppose that $\bar{v} \equiv 0$ in $\bar{\Omega}$. Then it follows from (6.1) and (6.2) that

$$
\lim _{i \rightarrow \infty}\left(a-u_{i}+c v_{i}\right)=a-\tau \mu \quad \text { and } \quad \lim _{i \rightarrow \infty}\left(-b+d u_{i}-v_{i}\right)=-b+d \tau \mu
$$

uniformly in $\Omega$. On the other hand,

$$
\int_{\Omega} u_{i}\left(a-u_{i}+c v_{i}\right) d x=\int_{\Omega} v_{i}\left(-b+d u_{i}-v_{i}\right) d x=0
$$

for all $i \in N$. Consequently, $a-\tau \mu=-b+d \tau \mu=0$ because of $u_{i}>0$ and $v_{i}>0$ in $\Omega$ and thus $a d-b=0$. This contradicts (1.1). Therefore $\bar{v}>0$ in $\bar{\Omega}$. By (6.1), (6.2) and (6.4), it is clear that

$$
\int_{\Omega}(\mu+\bar{v})\{a-\tau \mu+(c-\tau) \bar{v}\} d x=\int_{\Omega}(\mu+\bar{v})\{a-\tau(\mu+\bar{v})+c \bar{v}\} d x=0 .
$$

Hence it only remains to show $1<d \tau<b / \mu$. By the assumption of Theorem 1.3,

$$
-b+d \tau \mu<-\mu+d \tau \mu=\mu(d \tau-1) .
$$

It thus follows from Lemma 3.1 and (6.3) that if $d \tau-1 \leq 0$, then $\max _{\bar{\Omega}} \bar{v} \leq 0$ and this contradicts $\bar{v}>0$ in $\bar{\Omega}$. Therefore, $d \tau>1$. Using Lemma 3.1 and $\bar{v}>0$ in $\bar{\Omega}$ again, we obtain $d \tau<b / \mu$. Hence we complete the proof.

Acknowledgement. The author would like to express his gratitude to Professor Yoshio Yamada for his useful advice.

\section{References}

[1] Chen, B. and Peng, R., Coexistence states of a strongly coupled prey-predator model, J. Partial Differential Equations, 18 (2005), 154-166.

[2] Chen, X. F., Qi, Y. W. and Wang, M. X., A strongly coupled predator-prey system with non-monotonic functional response, Nonlinear Anal., 67 (2007), 1966-1979.

[ 3 ] Crandall, M. G. and Rabinowitz, P. H., Bifurcation from simple eigenvalues, J. Functional Analysis, 8 (1971), 321-340.

[4] Gilbarg, D. and Trudinger, N. S., Elliptic Partial Differential Equations of Second Order, 2nd Edition, Springer-Verlag, Berlin, 1983.

[5] Kadota, T. and Kuto, K., Positive steady states for a prey-predator model with some nonlinear diffusion terms, J. Math. Anal. Appl., 323 (2006), 1387-1401.

[6] Kuto, K., A strongly coupled diffusion effect on the stationary solution set of a prey-predator model, Adv. Differential Equations, 12 (2007), 145-172. 
[ 7 ] Lin, C. S., Ni, W. M. and Takagi, I., Large amplitude stationary solutions to a chemotaxis system, J. Differential Equations, 72 (1988), 1-27.

[ 8 ] Ling, Z. and Pedersen, M., Coexistence of two species in a strongly coupled cooperative model, Math. Comput. Modelling, 45 (2007), 371-377.

[9] Lou, Y., Nagylaki, T. and Ni, W. M., On diffusion-induced blowups in a mutualistic model, Nonlinear Anal., 45 (2001), 329-342.

[10] Lou, Y. and Ni, W. M., Diffusion, self-diffusion and cross-diffusion, J. Differential Equations, 131 (1996), 79-131.

[11] Lou, Y. and Ni, W. M., Diffusion vs cross-diffusion: an elliptic approach, J. Differential Equations, 154 (1999), 157-190.

[12] Nirenberg, L., Topics in Nonlinear Functional Analysis, American Mathematical Society, Providence, RI, 2001.

[13] Okubo, A. and Levin, L. A., Diffusion and Ecological Problems: Modern Perspective, 2nd Edition, Interdisciplinary Applied Mathematics, 14, Springer-Verlag, New York, 2001.

[14] Pang, P. Y. H. and Wang, M. X., Strategy and stationary pattern in a three-species predatorprey model, J. Differential Equations, 200 (2004), 245-273.

[15] Pao, C. V., Nonlinear Parabolic and Elliptic Equations, Plenum Press, New York, 1992.

[16] Shigesada, N., Kawasaki, K. and Teramoto, E., Spatial segregation of interacting species, J. Theoret. Biol., 79 (1979), 83-99.

[17] Turing, A. M., The chemical basis of morphogenesis, Philos. Trans. Roy. Soc. London B, 237 (1952), 37-72.

[18] Wang, M. X., Stationary patterns caused by cross-diffusion for a three-species prey-predator model, Comput. Math. Appl., 52 (2006), 707-720.

[19] Zhou, H. and Lin, Z., Coexistence in a strongly coupled system describing a two-species cooperative model, Appl. Math. Lett., 20 (2007), 1126-1130.

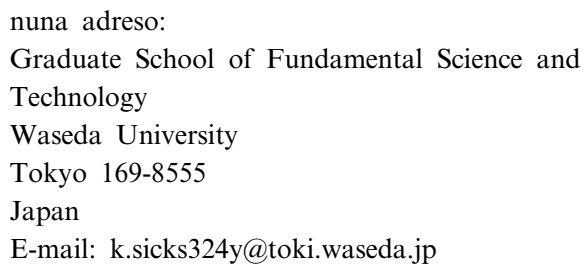

(Ricevita la 26-an de februaro, 2008)

(Reviziita la 12-an de majo, 2008) 